\title{
Parametric Analysis of Ultrasound Backscatter Signals for Monitoring Cancer Cell Structural Changes during Cancer TREATMENT
}

\author{
Harshitha Nallapareddy ${ }^{1}$, Sridhar Krishnan ${ }^{1}$ and Michael Kolios ${ }^{1,2}$ \\ ${ }^{1}$ Dept. of Elec. \& Comp. Engg., Ryerson University, Toronto, ON, Canada. \\ ${ }^{2}$ Dept. of Physics, Ryerson University, Toronto, ON, Canada. \\ (hnallapa)(krishnan)@ ee.ryerson.ca, mkolios@ryerson.ca
}

\begin{abstract}
High frequency ultrasound backscattered signals $(20-60 \mathrm{MHz})$ from normal and apoptotic cell pellets differ in their backscatter intensity, and analyzing these signals could assist in the non-invasive monitoring of cancer therapy. In this work, the reflection coefficients of the lattice prediction error filter are used as feature set for parametric analysis and signal classification. The ultrasound (US) backscattered signal databases consisted of combinations of treated (apoptotic) and untreated (normal) cells mixed in different proportions. A $40 \mathrm{MHz}$ commercial ultrasound imaging system was used. A classification accuracy of $97-100 \%$ for normal and apoptotic signals were obtained with a model order 15 . The positive results ascertain that the reflection coefficient is a potential tool for analyzing biomedical signals such as US backscattered signals.
\end{abstract}

\section{SOMMAIRE}

Les ultrasons à haute fréquence backscattereddes signaux (20 - 60 mégahertz) de normal et les granules apoptotic de cellules diffèrent dans leur intensité de rétrodiffusion, et l'analyse de ces signaux pourrait aider à la surveillance non envahissante de la thérapie de cancer. Dans ce travail, les coefficients de réflexion du filtre d'erreurs de prévision de trellis sont employés comme le dispositif a placé pour la classification paramétrique d'analyse et de signal. Les ultrasons (US) backscattered des combinaisons composées par bases de données de signal des cellules (normales) traitées (apoptotic) et non traitées mélangées dans différentes proportions. Un système commercial de formation image d'ultrasons de 40 mégahertz a été employé. Une exactitude de classification de 97-100\% pour les signaux normaux et apoptotic ont été obtenues avec un ordre modèle 15. Les résultats positifs établissent que le coefficient de réflexion est un outil potentiel pour analyser les signaux biomédicaux tels que les ultrasons backscattered des signaux.

\section{INTRODUCTION}

During the cell division if the DNA is not replicated properly, the cell stops the division cycle and kills itself. This self-induced destruction or programmed cell death is called as apoptosis. However, at times cells lose ability to kill themselves and their uncontrolled cell division forms a tumor potentially. Thus any dysfunction or deregulation in apoptosis process leads to cancer. Cancer is a term for diseases in which abnormal cells divide without any control and have the ability to invade nearby tissues and can spread through the blood stream and lymphatic system to other parts of the body. Here arises a need to suppress the fast and uncontrolled cell division: one way is to forcefully induce apoptosis.

To this end, many cancer treatments are developed including radiation therapy, chemotherapy, and immunotherapy to kill the cells by apoptosis or necrosis. At this point, it is desirable to have a technique that can detect apoptotic regions in an organ or tissue which is undergoing cancer treatment (e.g., chemotherapy) in order to ascertain the success of the treatment. At present, the evaluation of the cancer therapy is usually done by physical examination, assessing tumor shrinkage, and less frequently by imaging. This is usually done only after the patient undergoes the complete treatment cycle, which takes few weeks or months. There is an increasing need for a rapid therapy detection technique.

Various techniques (both invasive and non-invasive) have been developed to determine whether the cells are undergoing apoptosis. Biological techniques developed are invasive and time consuming as well. For example, Positron Emission Tomography (PET) though non-invasive, requires the injection of radioisotopes into the body and hence scans cannot be performed repetitively. Other optical imaging methods using bioluminescence markers, though non-invasive, lack penetration depth. It has been shown that high frequency ultrasound (HFUS) imaging in the range of 20-60 MHz can be used to detect the structural changes during the cell death [1]. 
HFUS imaging has become a powerful clinical tool and has proved especially valuable in ophthalmology. It has several advantages: it is non-invasive, non-ionizing, provides finescale resolution (especially for small animal anatomy), cost effective, safe and could be used to detect and identify a wide variety of abnormal tissues.

A series of structural changes occur within a cell during cell death. During apoptosis there is cell and nucleus shrinkage, condensation of chromatin in the nucleus, and eventual nuclear fragmentation. Studies over the past decade have found that the HFUS (20-100 MHz) can be used to detect localized variations in cell morphologies in tissues and cell ensembles [1,2]. It is shown in [2] that ensembles of necrotic (heat killed), cells undergoing mitosis, and apoptosis yield an increased backscatter ultrasound signal intensity compared to cells not exposed to the drug. US backscatter signals from normal and apoptotic acute myeloid leukemia (AML) cell pellets are shown in Figure 1. Spectral analysis techniques have been used to analyze radio frequency (RF) echo signals, and have made it possible to more specifically characterize average cell structure changes in tissues and cell ensembles. However, to increase the technique sensitivity and specificity, other signal analysis techniques are explored.

Farnoud [4], using Burg-lattice based autoregressive (AR) modeling successfully classified 100 backscatter signals from normal and apoptotic cells using machine-learning algorithms with a classification accuracy ranging from 50\%$97 \%$ with different classifiers. It was shown that non-linear classifiers such as probabilistic neural networks with sigmoid activation function provided the best accuracy. Bejar [5] could monitor apoptosis by using cepstral coefficients (derived from $\mathrm{AR}$ coefficients) as features and local discriminant bases (LDB) algorithm. This work used 39 signals of the normal group and 36 signals of the abnormal group and achieved an overall classification accuracy rate of above $90 \%$. Reflection coefficients, the parameters of the lattice filter, provide an alternative parameterization of signals. The reflection coefficients are computed from AR coefficients by using Levinson's recursions. There is a non-linear relation between these two coefficients. In this work we explore whether the reflection coefficients can potentially be used as signal features for the classification. With this motivation, we intend to find whether reflection coefficients may contain useful information about the US backscattered signal in such a way that the classification of the normal and apoptotic signals can be done by using simple and efficient time-domain pattern analysis approaches. To the author's best knowledge, this is the first study exploring the use of reflection coefficients for a biomedical signal classification application.

The block diagram of the proposed system is shown in Figure 2. The US backscattered signals are analyzed by using lattice prediction error filter parameters called reflection coefficients as features and classified by using simple classifier based on maximum likelihood method. The paper is structured as follows: Section 2 details the lattice prediction error filters, different algorithms to compute reflection coefficients including the Burg-lattice method. Results are discussed in
Section 3, and conclusions are given in Section 4.

\section{METHODOLOGY}

\subsection{Lattice Prediction Error Filter}

The objective of a linear prediction filter is to select a linear function that minimizes the prediction error for the given data set. When the predictor is embedded in the linear filter, the predictor can be viewed as linear filtering and is called as prediction error filter (PEF).

The PEF is defined as a structure, which combines successive samples of a signal multiplied by coefficients, so that the output (prediction-error) power of the filter is minimized. There are two kinds of PEF, depending on the form of prediction error utilized. Based on a given sequence of input samples, a forward PEF is designed to minimize the meansquare value of the forward prediction error, defined as the difference between the predicted value of the input one step into the future and its actual value. On the other hand, a backward PEF is designed to minimize the mean-square value of the backward prediction error, defined as the difference between the predicted value of the input one step into the past and its actual value.

Two basic adaptive filtering implementation schemes of the prediction error filter are the tapped-delay-line (TDL) structure, which is adapted by minimizing a single, global error criterion, and lattice structure, in which the error is minimized independently for each stage of the filter. Depending on the form of calculation used, the PEF may suffer from lack of numerical stability. The lattice PEF, a form of adaptive filter, proposed by Burg [6] and independently derived by Itakura and Saito [7] provides a solution to these problems. Lattice-structure has a number of advantages [8] over the traditional TDL structure, among which are better resolution and/or stability, much better control of the filter convergence and adaptive properties (due to the orthogonalization of the data provided by the lattice, the adaptive convergence rate appears to be particularly insensitive to the conditioning or eigenvalue spread of the input signal sequence), the stage by stage approach to the estimation problem provided by the lattice filter offers the possibility of determining the optimal model order for the process. The sensitivity of the lattice filter parameters to round off noise and finite word length effects, particularly in the normalized algorithms, seems to be less than that of the equivalent TDL processor. Some important characteristics of the lattice filters are [9]:

1. It is an efficient structure for generating simultaneously the forward and backward prediction errors.

2. The lattice structure is modular: increasing the order of the filter requires adding only one extra module, leaving all other modules and its associated filter parameters the same.

3. The various stages of a lattice are decoupled from each other in the following sense: The memory of the lattice (storing $b_{0}(n-1), \ldots, b_{m-1}(n-1)$ ) contain orthogonal

Vol. 35 No. 2 (2007) - 48 


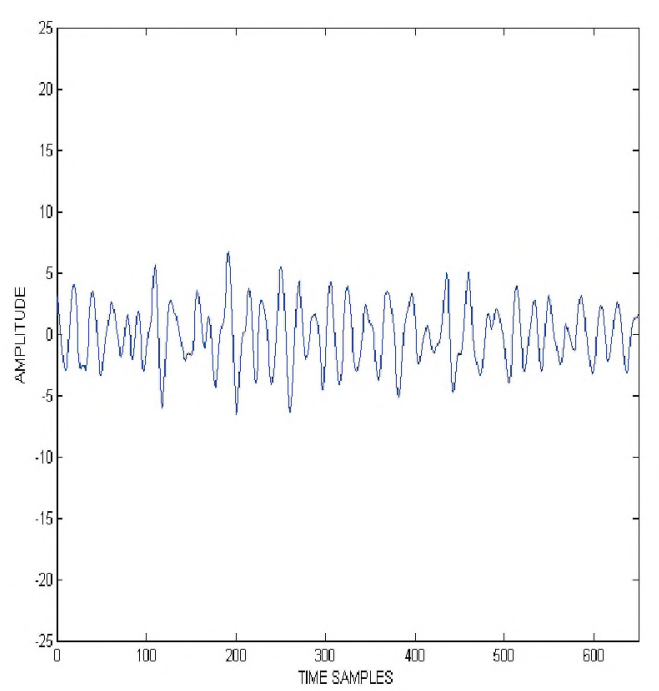

(a)

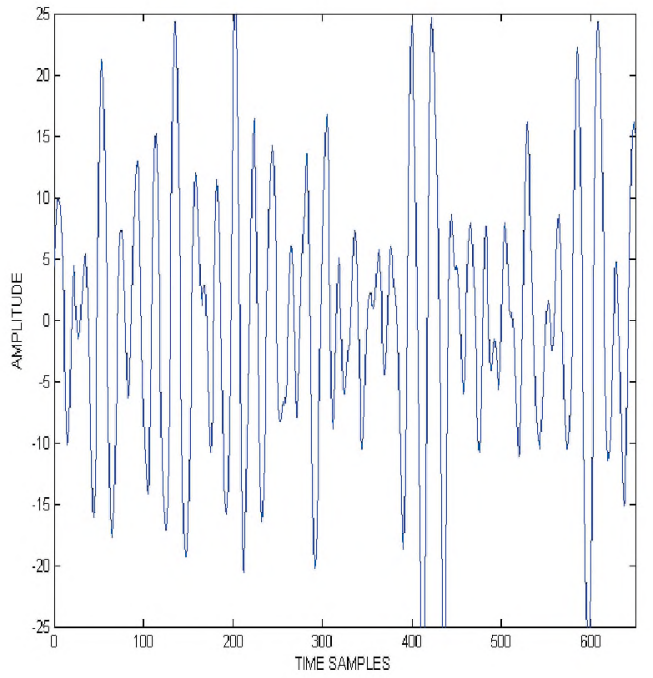

(b)

Figure 1: (a) Normal signal and (b) Apoptotic signal acquired from cell pellets.

variables, thus the information contained in $u(n)$ is split in $M$ pieces, which gradually reduces the redundancy of the signal.

The lattice structure, with superior flexibility and structural diversity find use in applications such as predictive filtering [3], adaptive filtering [10], and speech processing [15]. The lattice form filter realization first attracted great attention in the late 1960s and early 1970s, with its superiority in finite precision performance. Itakura and Saito were the first researchers who utilized this lattice form for speech coding.

\subsection{Algorithms for computing reflection coeffi- cients}

Lattice filters are a well-known signal analysis and coding tool. Their parameters, the reflection coefficients, have a good robustness to noise and quantization effects [16]. In the lattice formulation, the reflection coefficients can be computed by minimizing the norm of the forward residual or the backward residual, or a combination of the two. There are several methods to calculate the reflection coefficients of a lattice filter [11]. These methods depend on different ways of correlating the forward and backward residuals. A brief description of four of these algorithms is given below:

The common basic objective of all the algorithms mentioned is to minimize the mean-squared forward and backward errors, which are the outputs of each filter stage. In other words, to obtain the lowest values of $F_{i}(n)$ and $B_{i}(n)$, defined in the following equations:

$$
\begin{aligned}
& F_{i}(n)=E\left[\left|f_{i}(n)\right|^{2}\right] \\
& B_{i}(n)=E\left[\left|b_{i}(n)\right|^{2}\right]
\end{aligned}
$$

where $f_{i}(n)$ is the forward residual, $b_{i}(n)$ is the backward residual and $E($.$) denotes the expected value.$

Differentiating these quantities with respect to the reflection coefficient gives two values for the coefficient, by minimizing the forward and backward mean square errors separately. The equation

$$
\rho_{i}^{F}(n)=\frac{C_{i-1}(n)}{B_{i-1}(n-1)}
$$

minimizes the forward error, and

$$
\rho_{i}^{B}(n)=\frac{C_{i-1}(n)}{F_{i-1}(n)}
$$

minimizes the backward error where $C_{i}(n)$ is the expectation of the negative cross-power of forward and backward errors, given by

$$
C_{i-1}(n)=-E\left[f_{i}(n) \cdot b_{i}^{*}(n-1)\right]
$$

\subsubsection{Forward-and-Backward (F+B) Algo- rithm}

This is the most direct method suggested by Griffith [9] and is the only algorithm where the forward and backward reflection coefficients are not complex conjugates of each other. It simply uses $\rho_{i}^{f}(n)$ and $\rho_{i}^{b}(n)$ as the forward and backward reflection coefficients respectively, i.e.,

$$
\begin{aligned}
& \rho_{i}^{f}(n)=\rho_{i}^{F}(n) \\
& \rho_{i}^{b}(n)=\rho_{i}^{B}(n)
\end{aligned}
$$

As $\rho^{F} \cdot\left(\rho^{B}\right)^{*}=1$ in almost all cases either or will be greater than one, however, the reflection coefficients should 


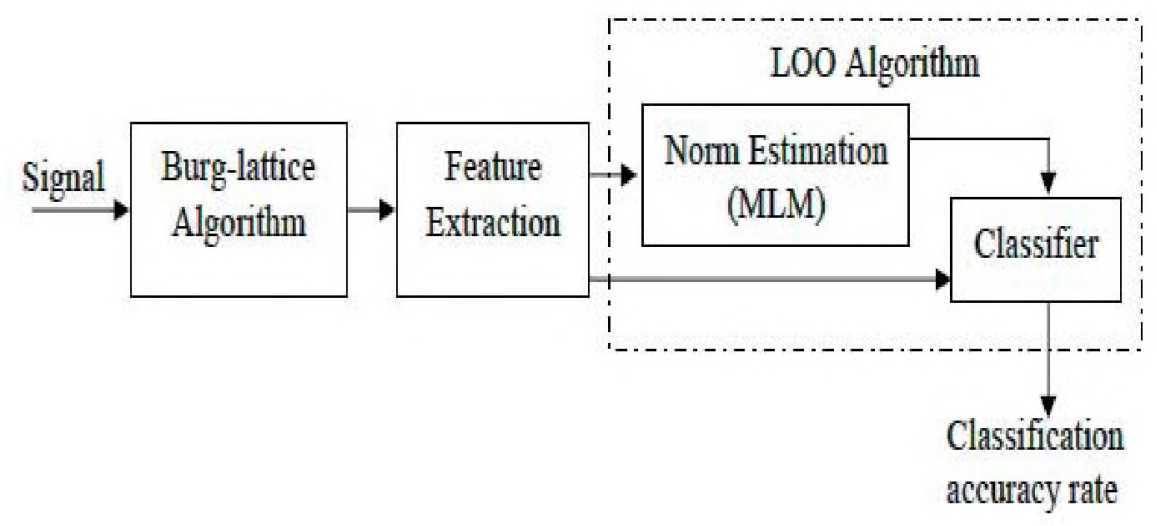

Figure 2: Block diagram of the proposed system

have a value less than one for a stable filter. Hence, the stability is not guaranteed.

\subsubsection{Forward/Backward-Minimum (M) Al- gorithm}

This algorithm provides an alternative to the $\mathrm{F}+\mathrm{B}$ algorithm by following the rule that if either $\rho_{i}^{F}(n)$ or $\rho_{i}^{B}(n)$ is greater than one, then the other will be less than one and hence guarantees stability. This algorithm is suggested by Makhoul [13], and is formulated as

$$
\begin{gathered}
\rho_{i}^{f}(n)=\rho_{i}^{M}(n)=\frac{C_{i-1}(n)}{\max \left[F_{i-1}(n), B_{i-1}(n-1)\right]} \\
\rho_{i}^{b}(n)=\left[\rho_{i}^{M}(n)\right]^{*}
\end{gathered}
$$

\subsubsection{Geometric-Mean (G) Algorithm}

This algorithm is one of the two joint estimation algorithms that try to minimize the forward and backward error expectations jointly and is derived by Itakura and Saito [7]. Here, the reflection coefficients are computed by using the geometric mean of the forward and backward error expectation.

$$
\begin{gathered}
\rho_{i}^{f}(n)=\rho_{i}^{G}(n)=\frac{C_{i-1}(n)}{\left[F_{i-1}(n) \cdot B_{i-1}(n-1)\right]^{1 / 2}} \\
\rho_{i}^{b}(n)=\left[\rho_{i}^{G}(n)\right]^{*}
\end{gathered}
$$

\subsubsection{Burg Algorithm}

Burg method is an order- recursive algorithm and was introduced by J.P. Burg in 1967 [6]. This method uses a lattice filter and directly estimates reflection coefficients instead of autocorrelation values. The algorithm is sometimes designated as maximum entropy method because of its derivation in the context of maximum entropy methods. The key step in the algorithm involves minimizing the sum of the norm of the forward and backward residual vectors, as a function of the reflection coefficient matrices. Since the computed coefficients are the harmonic mean between the forward and backward partial autocorrelation estimates, the Burg procedure is also known as the Harmonic algorithm. This algorithm starts with a first-order model and computes the prediction parameters (reflection coefficients) for successively higher model orders. The ith reflection coefficient is a measure of the correlation between $y(n)$ and $y(n-i)$ after the correlation due to the intermediate observations $y(n-1), \ldots, y(n-i+1)$ has been filtered out. As the recursion constrains the filter poles to fall within the unit circle stability of the filter is gauranteed. The Burg method is particularly useful for estimating coefficients from segments of unequal length. This method is based on Levinsons recursions and estimates the $\mathrm{AR}$ filter parameters through the associated reflection coefficients constraining the AR coefficients to satisfy Levinson equations.

Lets assume the data measurements (US backscattered signals) be $\{y(n)\}$ for $n=0,1,2, \ldots, N-1$ and let us consider the filter of order $M$.

The equations of the Burg-lattice filter are:

$$
f_{0}(n)=b_{0}(n)=y(n)
$$

$$
\begin{aligned}
& f_{i}(n)=f_{i-1}(n)+k_{i} b_{i-1}(n-1), 1 \leq i \leq M \\
& b_{i}(n)=k_{i} f_{i-1}(n)+b_{i-1}(n-1), 1 \leq i \leq M
\end{aligned}
$$

The corresponding ith reflection coefficient $k_{i}$ is obtained by minimizing the sum of the square values of the forward and backward prediction errors at the output of the $i t h$ stage.

$$
k_{i}=\frac{-\sum_{n} f_{i-1}(n) b_{i-1}(n-1)}{\frac{1}{2} \sum_{n}\left[\left|f_{i-1}(n)\right|^{2}+\left|b_{i-1}(n-1)\right|^{2}\right]}
$$

As the Burg algorithm uses lattice structure, it inherits the advantages of lattice structure such as stability, modularity, computational simplicity and efficiency. Besides these, 


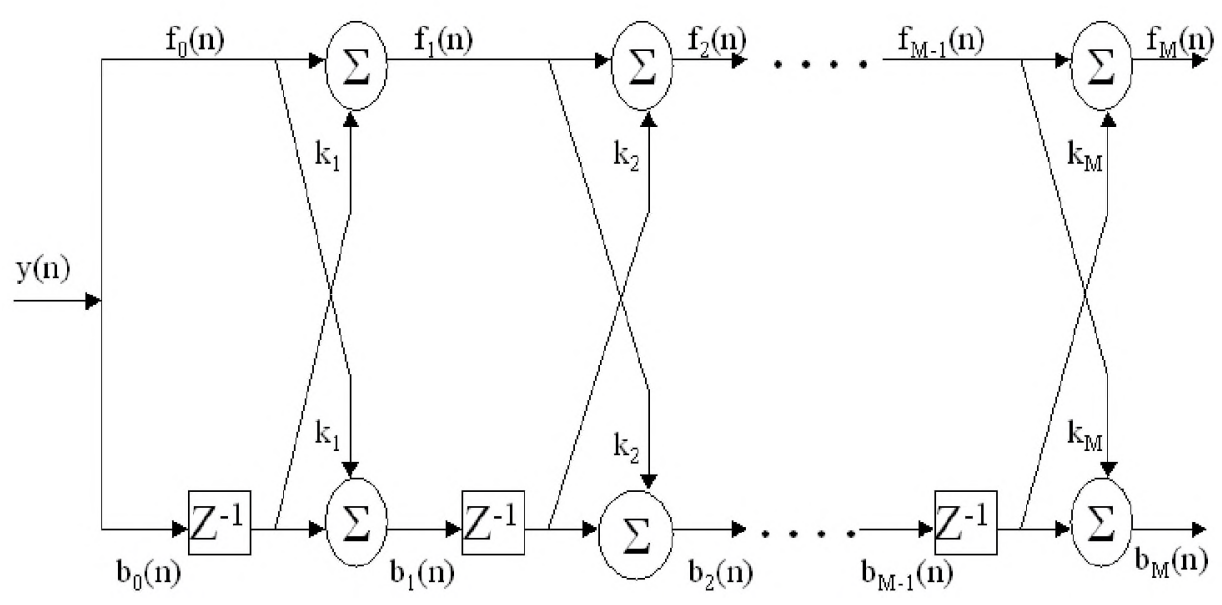

Figure 3: Burg-lattice Filter (adapted from [18])

it is proven to be an efficient linear prediction technique and is probably the most widely known method to estimate $A R$ coefficients. Considering the advantages of Burg-lattice algorithm, in this work its reflection coefficients are used for parametric feature representation of the ultrasound backscatter signals. We also experimentally checked the time invariance property and robustness of the reflection coefficients of the Burg algorithm. In time invariance test, reflection coefficients of an apoptotic signal and its delayed version $(0.2 \mathrm{mi}-$ cro seconds) are calculated. From Table 1 it can be demonstrated that the reflection coefficients are time invariant as the value of the reflection coefficients are same. In robustness test, an apoptotic signal is corrupted with random noise. The reflection coefficients for both apoptotic and noisy apoptotic signals are calculated. Then a measure of closeness of the two sets of reflection coefficients is calculated by using the correlation coefficient function. Table 2 shows the correlation coefficients obtained for different signal to noise ratio(SNR)s. The reflection coefficients are identical for a SNR of $30 \mathrm{~dB}$ and show strong correlations for lower SNRs $(>9 \mathrm{~dB})$.

\subsection{Data Acquisition}

AML-5 cells (at Ontario Cancer Institute) were cultured in alpha minimum essential medium (alpha MEM, Gibco 11900), supplemented with Streptomycin and Penicillin at concentrations of $100 \mathrm{mg} / \mathrm{L}$, and $5 \%$ Fetal Bovine serum ( Hyclone). The cells grew in $150 \mathrm{ml}$ of medium as a suspension, at concentrations of $5 \times 105$ cells $/ \mathrm{ml}$, in a $370 \mathrm{C}$, and $5 \% \mathrm{CO}_{2}$ incubator [19]. Pellets were made with untreated cells and treated cells. Treated cells were exposed to $10 \mathrm{ug} / \mathrm{ml}$ cisplatin, a chemotherapeutic agent for 24 hours, to induce apoptosis, before processing to form a pellet. Large volumes of the treated and untreated cells were concentrated by centrifugation, at 2000rpm for 10 minutes, using a Sorval centrifuge. The cell concentrations were then determined and volumes prepared in phosphate-buffered saline, so that the final pellets would have the desired percentages of the treated and untreated cells. The final pellets for scanning were centrifuged at $3000 \mathrm{rpm} / 10$ minutes, in flat bottom cryo-tubes on a desktop swinging bucket centrifuge. The cell pellets were then immersed in phosphate-buffered saline that acted as a coupling medium for the ultrasound imaging and RF data collection. During the data acquisition process, the cells were kept at room temperature. The experimental set consists of a pellet of the normal or untreated AML cells (which are not exposed to cisplatin drug), and different mixtures of treated and untreated cells. The mixtures of cells varied from 5\% treated cells mixed with $95 \%$ untreated to $100 \%$ treated cells. The cells are imaged as a function of concentration of treated cells $(5,10,20,80$ and $100 \%)$. A $40 \mathrm{MHz}$ f 2 transducer with a bandwidth of approximately $100 \%$ was used to image the pellets of normal and apoptotic cells. The transducer was attached to the VS40B ultrasound imager (Visualsonics Inc., Toronto, ON, Canada) which has the ability to select regions of interest (ROI) from the B-scan images and store the raw RF backscattered data of the ROI. The RF data was digitized at $500 \mathrm{MHz}$ sampling rate and stored for further analysis. Data analysis was performed in MATLAB (The Mathworks Inc., Natick, MA, USA). The experimental data were obtained in Princess Margaret Hospital, Toronto, Canada. Experimental details on the data acquisition can be found in [17].

\subsection{Feature Extraction and Classification}

The experimental ultrasound backscatter signals, like many other biomedical signals could be non-stationary. They are segmented into stationary segments in order to apply standard signal processing techniques such as parametric analysis. In the present work, manual fixed segmentation method is used. In the B-scan image as shown in Fig. 4, a small portion of about $1 \mathrm{~mm}$ at the centre of the image $(0.5 \mathrm{~mm}$ above and below the focal line of the transducer) is selected as the segment. The segment length is of 650 samples. The stationary (quasi-stationary) segments are then given to the lattice prediction error filter and the reflection coefficients are obtained by using the Burg-lattice algorithm. These reflection coefficients (partial correlation coefficients) are assumed 


\begin{tabular}{|l|l|l|l|l|l|}
\hline$k_{o}$ & -0.9459 & 0.9215 & 0.1836 & -0.2651 & -0.0626 \\
\hline$k_{d}$ & -0.9459 & 0.9215 & 0.1836 & -0.2651 & -0.0626 \\
\hline
\end{tabular}

Table 1: Reflection coefficients of original and delayed apoptotic signal. $k_{o}$ represents reflection coefficients of original apoptotic signal and $k_{d}$ represents reflection coefficients of the delayed

\begin{tabular}{|c|c|c|c|c|c|c|c|c|c|c|c|}
\hline SNR (in dB) & 0 & 3 & 6 & 9 & 12 & 15 & 18 & 21 & 24 & 27 & 30 \\
\hline $\begin{array}{c}\text { Correlation } \\
\text { Coefficients }\end{array}$ & 0.30 & 0.38 & 0.46 & 0.54 & 0.65 & 0.76 & 0.85 & 0.91 & 0.95 & 0.97 & 1.00 \\
\hline
\end{tabular}

Table 2: Correlation coefficient values of the original apoptotic signal and corrupted apoptotic signal with random noise at different SNRs

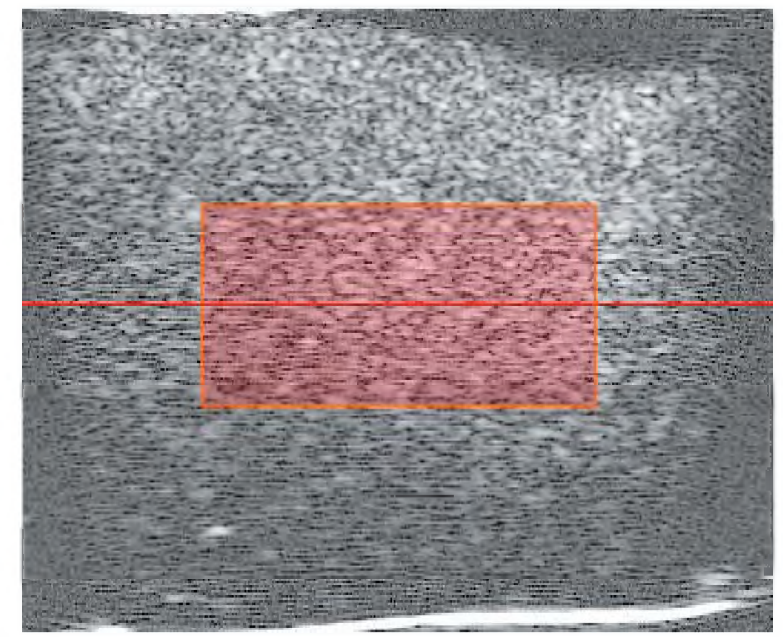

Figure 4: Segmentation The central line indicates the focal line of the transducer and the rectangle indicated the selected region of the image.

to have the discriminant statistical information of the signals and were treated as features. Model order selection is important. With appropriate number of poles, it is possible to reconstruct the signal. Typically, model order is twice the number of spectral peaks of the signal. In this work, the peaks were between 5 and 7 . Hence model order of 15 is selected. A snapshot of reflection coefficients of normal and apoptotic signal after 24 hours with a model order 15 is given in the Fig. 5.

Pattern classification is the next step after feature extraction in the pattern recognition process. As indicated in [14] the four best-known approaches for pattern recognition are: 1) template matching, 2) statistical classifications, 3) syntactic or structural matching, and 4) neural networks. In statistical pattern recognition, each pattern is represented by a set of $d$ features i.e., viewed as a $d$ dimensional vector. When little prior knowledge about the patterns to be recognised is known, the best suitable design for the pattern recognition system is to use training or a learning procedure. The classification is operated in two modes: training (learning) and testing (classification). The classifier is first trained with the derived features and then tested. Standard statistical classification methods use descriptive parameters and distance measures using probablistic approaches. There are several distance measures that could be used [5]: Euclidean distance measure, maximum likelihood measure, Mahalanobis distance measure, and weighted distance measure. In this present work, the classification of the ultrasound signals was done by using the maximum likelihood method as it most closely approximates the Bayes classifier and obtains the best discriminative efficiency if the probability density function of the extracted features are multivariate Gaussian $[5,14]$.

The classification accuracy is estimated by using leaveone-out (LOO) method, one of the most popular validation techniques. The LOO method is known to provide least bias estimate [14]. In this method, one sample is excluded from the dataset and the classifier is trained with the remaining samples. Then the classification accuracy is determined by testing the classifier with the excluded sample. This is repeated for all samples of the dataset. An independence between the test and the training set is maintained as each sample is excluded from the training set. The reference database consists of two template reference vectors (one for normal and the other for apoptotic signals). A test signal is extracted from the database, the distance between the test signal and the group of reference is measured. The test signal belongs to the group which has less norm.

\section{RESULTS AND DISCUSSION}

Each pellet data consists of $43 \mathrm{RF}$ lines collected from a $40 \mathrm{MHz}$ transducer. As mentioned before, the ultrasound backscattered signals from untreated cancer cells are termed as normal signals and those from the cancer cells treated with chemotherapeutic agent (which induces apoptosis) are termed as apoptotic signals. The treated cancer cells are imaged as a function of concentration of treated cells $(5,10,20,80$ and $100 \%$ ). The aim is to classify the signals at different concentrations of the treated cells. Statistical analysis of the signals helps in extracting the discriminative features.

All the signals from a database are fed as input to the Burg-lattice filter. The corresponding reflection coefficients are extracted as features. The classifier is trained with the extracted features and tested with an unknown signal. The classification results are tabulated. We compared the results obtained by using reflection coefficients as features with the results obtained by using AR coefficients and cepstral coeffi- 


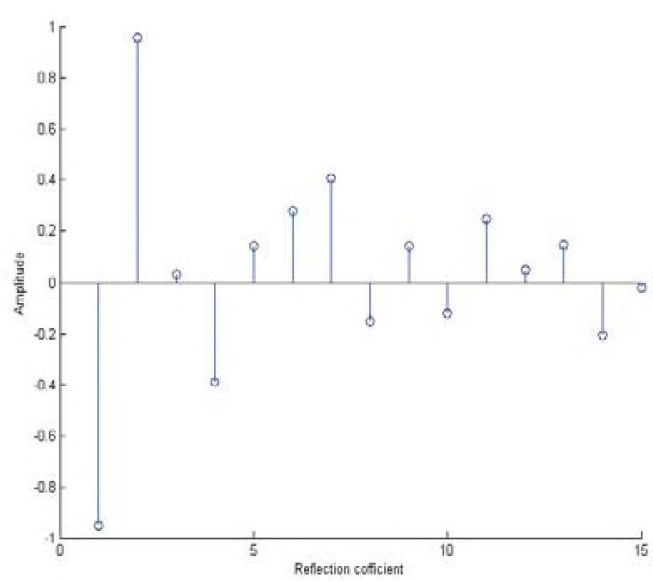

(a)

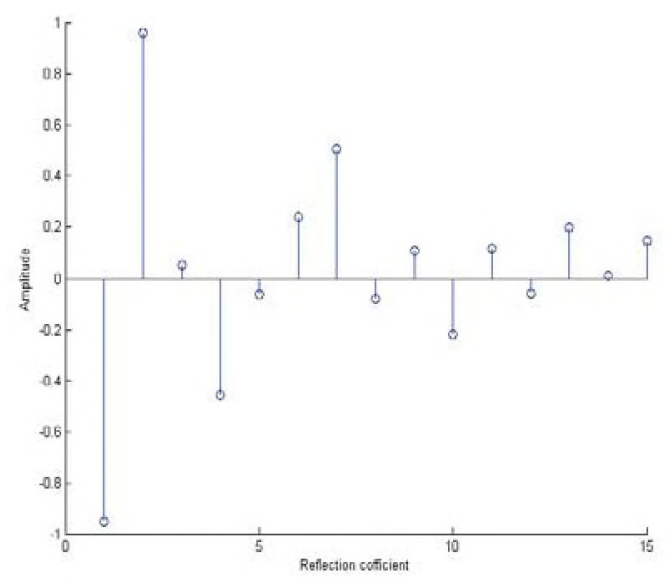

(b)

Figure 5: (a). A snapshot of reflection coefficients of signal from normal cells with a model order 15 and (b). A snapshot of reflection coefficients of signal from apoptotic cells (after $\mathbf{2 4}$ hours of exposure to cisplatin) with a model order of 15.

\begin{tabular}{|c|c|c|c|c|c|c|c|c|c|}
\hline \multirow[t]{2}{*}{$\begin{array}{l}\% \text { of treated } \\
\text { cells in pellets }\end{array}$} & \multicolumn{3}{|c|}{$\begin{array}{l}\text { Accuracy rate of } \\
\text { Normal Signals in } \\
\%\end{array}$} & \multicolumn{3}{|c|}{$\begin{array}{l}\text { Accuracy rate of } \\
\text { Apoptotic } \\
\text { in \% }\end{array}$} & \multicolumn{3}{|c|}{$\begin{array}{l}\text { Overall classifi- } \\
\text { cation accuracy in } \\
\%\end{array}$} \\
\hline & $\mathbf{R C}$ & $\overline{\mathbf{A R}}$ & Ceps & $\mathbf{R C}$ & $\overline{\mathbf{A R}}$ & Ceps & $\mathbf{R C}$ & $\mathbf{A R}$ & Ceps \\
\hline 5 & 100 & 83.72 & 95.35 & 100 & 88.37 & 97.67 & 100 & 86.05 & 96.51 \\
\hline 10 & 100 & 88.37 & 97.67 & 100 & 90.69 & 100 & 100 & 89.54 & 98.83 \\
\hline 20 & 97.67 & 83.72 & 88.37 & 97.67 & 86.05 & 90.70 & 97.67 & 84.88 & 89.54 \\
\hline 80 & 100 & 88.37 & 97.67 & 100 & 97.67 & 100 & 100 & 93.02 & 98.83 \\
\hline 100 & 100 & 88.37 & 97.67 & 100 & 95.35 & 100 & 100 & 91.86 & 98.83 \\
\hline
\end{tabular}

Table 3: Classification accuracy with model order 15. RC-reflection coefficients, AR- autoregressive coefficients and Ceps-cepstral coefficients

cients as features. The percentage represents the number of signals classified accurately. The overall classification represents the number of normal and apoptotic signals classified accurately.

Tables 3 show the classification accuracy rates obtained by comparing the normal signals with apoptotic signals at 5 , $10,20,80$, and $100 \%$ concentrations with a model order of 15. The classification accuracy rates indicate that the relfection coefficients provide the best classification of ultrasound backscattered signals. The cepstral coefficients give better performance than the AR coefficients. The reason could be the following discussion. AR coefficients gives a relatively abstract form of feature representation. However, cepstral coefficients are well suited for signals that contain echos of a fundamental signature (ultrasound backscatter could be cosidered as a signal resulting from a convolution of the pulse sent (fundamental signature) with the scattering strength of the scatterers) and hence may be better than AR. On the other hand, reflection coefficient is also an abstract parameter and for signals with reflected components, it might do a better feature representation. The better performance may be due to higher discriminant information being present in the derived reflection coefficients from the ultrasound backscattered signals from the normal and the apoptotic cells

\section{CONCLUSIONS}

In this paper we evaluated the accuracy of lattice filter prediction coefficients to differentiate the ultrasound backscatter signals from normal and apoptotic cells which differ in their intensity and frequency spectrum. This is the first work in biomedical signal analysis, in which reflection coefficients are used for parametric signal analysis and classification. The positive results, demonstrate the potential discriminatory ability by using reflection coefficients as features and are worth studying. Modularity, the main advantage of lattice structure, will make hardware implementation straight forward. From a practical perspective, the lattice provides an efficient, fast, modular and robust structure suitable for hardware implementation and hence it can widen the scope of research on the use of reflection coefficients.

Although this work has been focused mainly on evaluating the reflection coefficients to contain discriminant information about normal and apoptotic signals, further work on this "hardware-friendly" DSP technique will be aimed at 
evaluting the performance of reflection coefficients by testing with a larger database in real-time. Successful real-time performance will allow to reach the ultimate goal ie., hardware implementation and even extend its applications in analyzing other biomedical signals.

\section{REFERENCES}

[1] S. Brand, "Visualization of apoptotic cells using scanning acoustic microscopy and high frequency ultrasound", IEEE Symposium on Ultrasonics, vol 2, pp. 882-5, Sept. 2005.

[2] Kolios, M. C., et al., "An investigation of backscatter power spectra from cells, cell pellets and microspheres", IEEE Symposium on Ultrasonics, vol 1, pp. 752-7, Oct 2003.

[3] S.J. Ovaska, "Predictive Signal Processing in Instrumentation and Measurement: A Tutorial Review", IEEE Conference Proceedings on Instrumentation and Measurement Technology Conference, vol. 1, pp. 48 53, May 1997.

[4] Noushin R. Farnoud, Michael Kolios and Sridhar Krishnan, "Ultrasound Backscatter Signal Characterization and Classification Using Autoregressive Modeling and Machine Learning Algorithms", IEEE Conference Proceedings on Engineering in Medicine and Biology Society, vol. 3, pp. 2861 - 2864, Sep 17-21, 2003.

[5] Bejar, Jose. M. C., "Cepstral Analysis of Ultrasound Backscattered Signals for Screening Apoptotic Cancer Cells from Normal Cells", Ryerson University, Aug 2003.

[6] J. P. Burg, "Maximum Entropy Spectral Analysis", Ph.D. dissertation, Dept. of Geophysics, Stanford University, Stanford, CA, May 1975.

[7] F.Itakura and Saito, "Digital Filtering Techniques for Speech Analysis and Synthesis", Pesented at 7th Int. Cong. Acoustics, Budapest, paper 25-C-1, 1971.

[8] J. Makhoul, "Linear Prediction: A Tutorial Review", Proc. IEEE, Vol. 63, Issue 4, pp. 561-580, 1975.

[9] L. Griffiths, "A continuously-Adaptive Filter Implemented as a Lattice Structure", Proc. IEEE Int. Conf. on Acoustics, Speech, and Signal Processing, vol. 77CH1197-3, pp. $683-$ 686, 1997.

[10] Simon Haykin, "Adaptive Filter Theory", Prentice Hall Information and System Sciences Series, New Jersey, 2002.

[11] C.J. Gibson, Simon Haykin, "A Comparision of Algorithms for the Calculation of Adaptive Lattice Filters", IEEE International Conference on Acoustics, Speech, and Signal Processing, Vol 5, pp. 978-983, 1980.

[12] Sridhar Krishnan, "Adaptive Filtering, Modeling, and Classification of Knee Joint Vibroarthrographic Signals", Masters thesis, Department of Electrical and Computer Engineering, University of Calgary, Calgary, AB, Canada, April 1996.

[13] J. Makhoul, "Stable and Efficient Lattice Methods for Linear Prediction", IEEE Trans. on Acoustics, Speech, and Signal Processing, vol. ASSP-25, pp. 423-428,1977.

[14] Anil K. Jain et al., "Statistical Pattern Recognition: A Review", IEEE Transactions on Pattern Analysis and Machine Intelligence, Vol. 22, No. 1, Jan 2000.

[15] H. K. Kwan, M. Wang, "ARMA Lattice Model for Speech Analysis and Synthesis", Proc. IEEE International Conference on Neural Networks and Signal Processing, Vol 2, pp. $912-$ 915, Dec 2003.

[16] S. Krstulovic, F. Bimbot, "Signal modeling with non-uniform topology lattice filters", IEEE Proceedings on Acoustics, Speech, and Signal Processing, Vol. 2, pp. 845-848, May 2001.

[17] Adam S. Tunis, "Monitoring Structural Changes in Cells and Tissues with High Frequency Ultrasound Signal Statistics", M.Sc., University of Toronto (Canada), 2005.

[18] K. Roth, et al., "Frequency warped Burg's method for ARmodeling", IEEE Workshop on Applications of Signal Processing to Audio and Acoustics, pp. 5-8, Oct 2003.

[19] Czarnota G., Kolios M., Abraham J., Portnoy M., Ottensmeyer F., Hunt J. W. and Sherar M. ,'Ultrasonic Imaging of apoptosis:High-Resolution Non-invasive Imaging of Programmed Cell Death in vitro, n situ and in vivo", British Journal of Cancer 81(3), pp.520-527, 1999. 